\title{
Measurement of the UE activity in pp collisions with the CMS detector
}

Tomasz Frueboes $^{* \dagger}$ on behalf of the CMS collaboration

National Centre for Nuclear Research

E-mail: fruboes@cern.ch

\begin{abstract}
A review of the recent measurements of the underlying event activity in proton-proton collisions performed by CMS collaboration at different center of mass energies is presented. Comparisons of the measured data with predictions obtained using the underlying event models implemented in different MC generators show good agreement for basic UE properties, while differences show up in more detailed comparisons.
\end{abstract}

XXII. International Workshop on Deep-Inelastic Scattering and Related Subjects,

28 April - 2 May 2014

Warsaw, Poland

\footnotetext{
*Speaker.

†Supported by the "Mobility Plus" program of Ministry of Science and Higher Education, Republic of Poland
} 


\section{Introduction}

The Underlying Event (UE) in proton-proton collision is an irreducible background for many analyses. It is defined as all the activity resulting from the collision besides the hard interaction, including the products of Initial and Final State Radiation (ISR and FSR respectively), Multi Parton Interactions (MPI) and Beam-Beam Remnants [1].

In order to study UE properties one needs to disentangle the products of the hard process and the remaining particles. Typically in UE analyses the hardest object (present in a given event) is the reference object, where its exact definition depends on the analysis - it can be a leading track, a leading jet, a reconstructed lepton pair, etc. The reference object defines the event scale (typically the $p_{\mathrm{T}}$ of the reference object) and the direction (typically the direction of the reference object in the transverse plane). This direction allows to define three regions: "towards" $\left(|\Delta \phi|<60^{\circ}\right)$, "away" $\left(|\Delta \phi|>120^{\circ}\right)$ and "transverse" $\left(60^{\circ}<|\Delta \phi|<120^{\circ}\right)$. By definition the towards region contains products of the hard interaction. The away region is also affected by the hard interaction from the recoil of the hardest object. For UE studies the most interesting region is the transverse region, where the average $p_{\mathrm{T}}$ of particles or the average charged particle multiplicities are investigated. These quantities are typically studied as a function of the event scale.

\section{Studies of the Underlying Event activity using leading tracks}

The measurement of the UE properties as a function of the leading tracks [2] follows the analysis strategy described above. All charged tracks with $p_{\mathrm{T}}>0.5 \mathrm{GeV}$ and $|\eta|<0.8$ are considered. The reference object is the highest $p_{\mathrm{T}}$ (leading) track in the event. The average transverse momenta and the multiplicities of the charged particles in the transverse region are studied as a function of the transverse momentum of the leading tracks.

The study was performed with data recorded by CMS in p-p collisions at center-of-mass energies 0.9 and $7 \mathrm{TeV}$. Fig. 1 shows the comparison of the measurement (fully corrected for detector inefficiencies) with predictions obtained from different MC generators - Herwig++ [3, 4] (using the default UE tune), PYTHIA6 [5] (using Z1 and D6T UE tunes) and PYTHIA8 [6] (using tune1). Predictions with UE tunes Z1 and Tune 1 show the best compatibility to data. Predictions from Herwig++ fail to describe the data. It is worth noting that the Herwig++ tune was not obtained using measurements from LHC data. A comparison with more recent tunes can be found in [7], where a better agreement of predictions with the measurement is observed.

\section{Strange Particle Production in the Underlying Event}

The analysis of strange particle production in the UE [8] follows a similar approach, but requires identified particles. The average multiplicities and average transverse momenta of strange particles $\left(\Lambda\right.$ and $\left.K_{S}^{0}\right)$ are measured in the transverse region with respect to a leading jet built using charged particles. Measurements performed at $\sqrt{\mathrm{s}}=7 \mathrm{TeV}$ center-of-mass-energy are compared to predictions obtained with D6T, Z1, Z2* PYTHIA6 tunes and PYTHIA8 Tune 1 (Fig. 2). All predictions show significant differences to the measurement, with $\Lambda$ particles showing the largest discrepancy. 

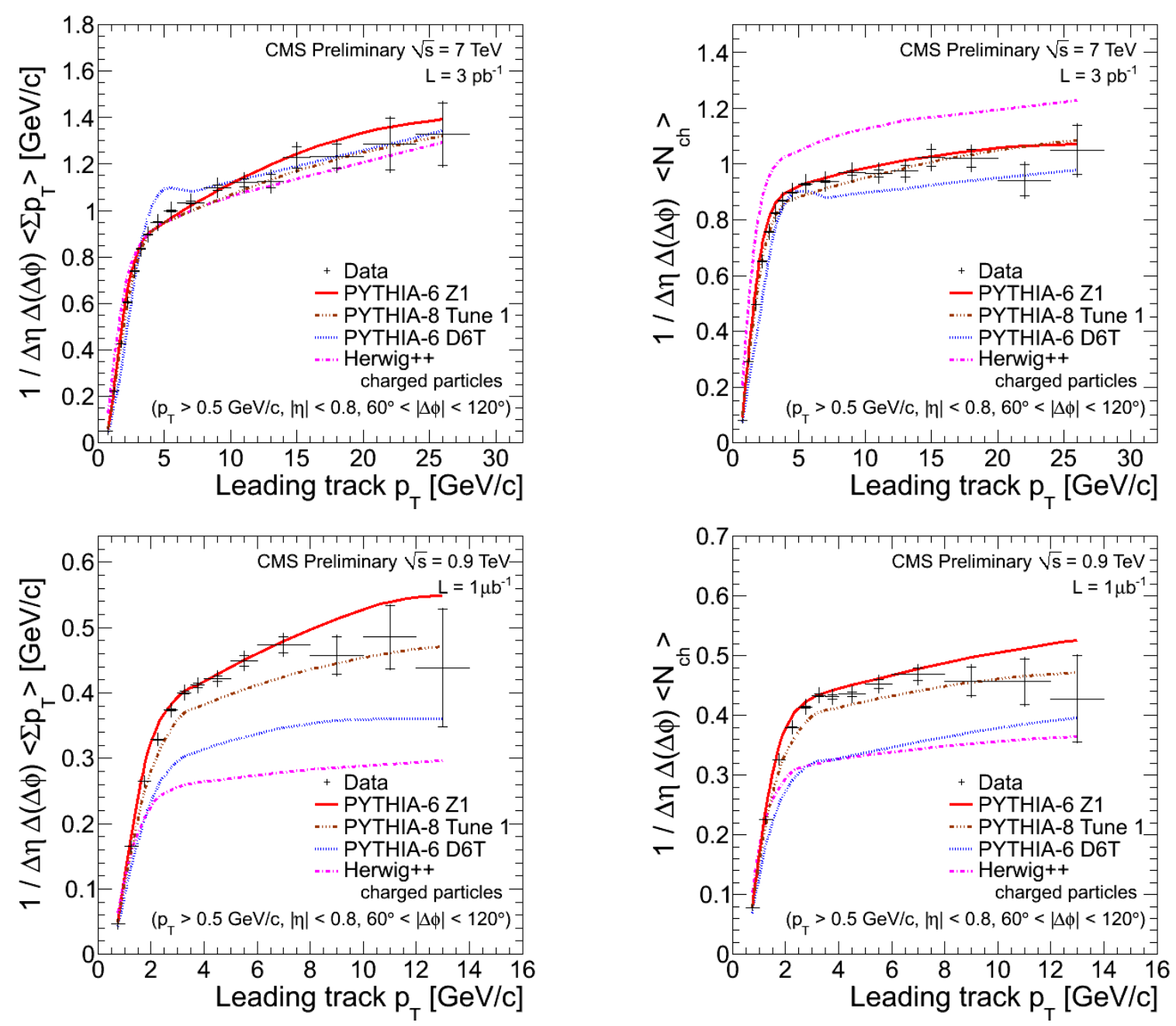

Figure 1: Comparison of the Monte Carlo predictions and fully corrected distributions of the average transverse momenta (left) and average particle multiplicities (right) in the transverse region as a function of the leading tracks $p_{\mathrm{T}}$. Results are shown for $7 \mathrm{TeV}$ (top) and $0.9 \mathrm{TeV}$ (bottom) center of mass energies.

\section{Underlying Event properties as a function of particle multiplicity}

It is also possible to distinguish between the hard interaction and the UE constituents using a different method as described in [9], where reconstructed tracks with $p_{\mathrm{T}}>0.25 \mathrm{GeV}$ and $|\eta|<2.4$ are clustered using the anti- $k_{t}$ algorithm. All tracks clustered into a jet with $p_{\mathrm{T}}>5 \mathrm{GeV}$ ("intrajet" tracks) are considered to be a product of the hard interaction. Tracks that fail this criterion ("soft" tracks) are considered being part of the UE. The distance parameter (equal to 0.5) of the anti- $k_{t}$ algorithm was set such that the momentum spectrum of the UE tracks does not change. Properties of the UE and the hard interaction are then studied in bins of multiplicity, which correspond to different event centralities.

The study was performed using $3.18 \mathrm{pb}^{-1}$ of data recorded at $\sqrt{s}=7 \mathrm{TeV}$ under low pileup conditions. The results are compared to predictions from PYTHIA6 (Z2* tune), PYTHIA8 (4C tune), Herwig++ 2.5 (tune UE_EE_3M) as well as with PYTHIA8 without MPI. All measured 

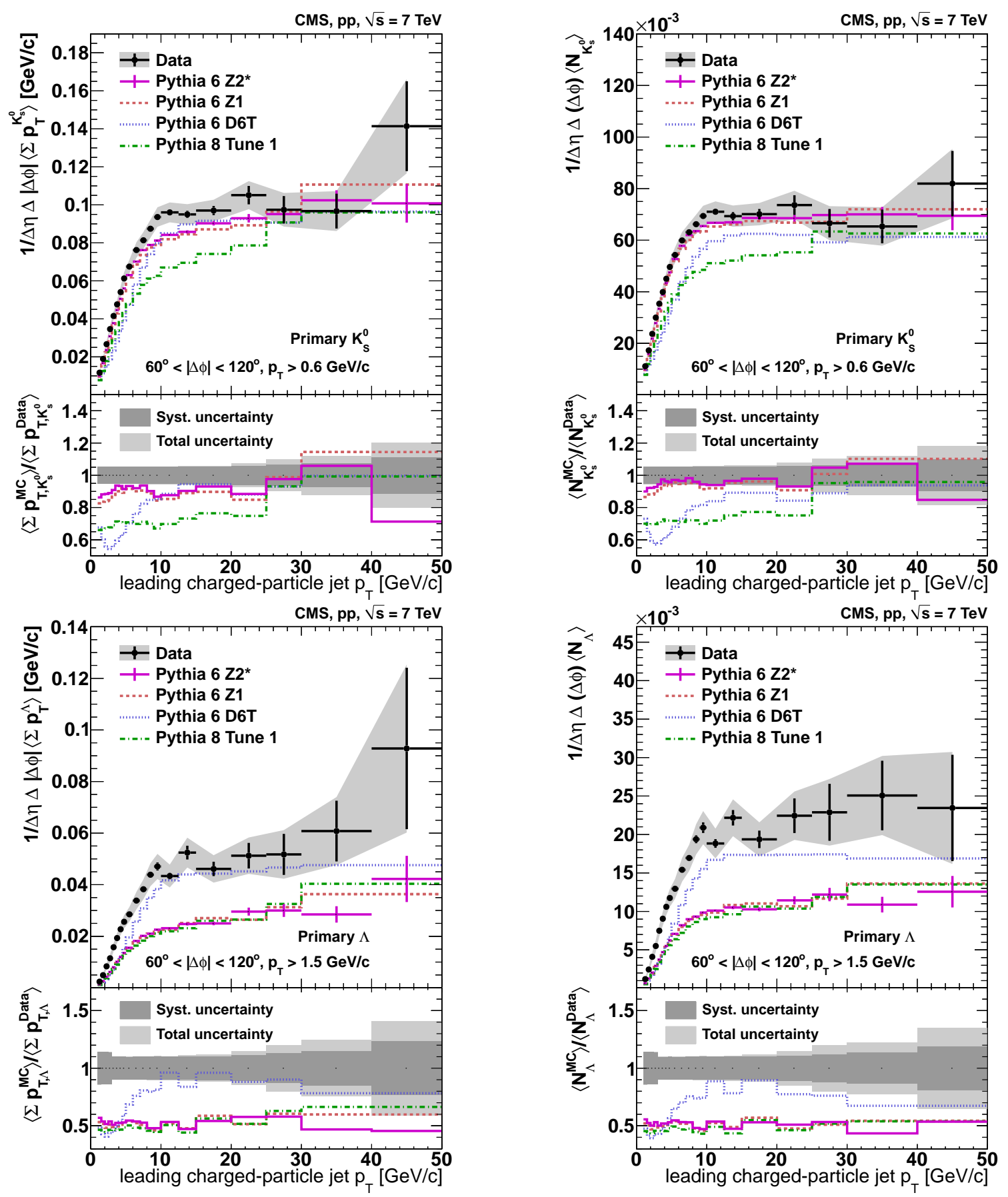

Figure 2: Comparison of the Monte Carlo predictions and fully corrected observed distributions of the average transverse momenta (left) and average particle multiplicities (right) in the transverse region as a function of the leading tracks transverse momentum for $K_{S}^{0}$ (top) and $\Lambda$ (bottom) particles. 

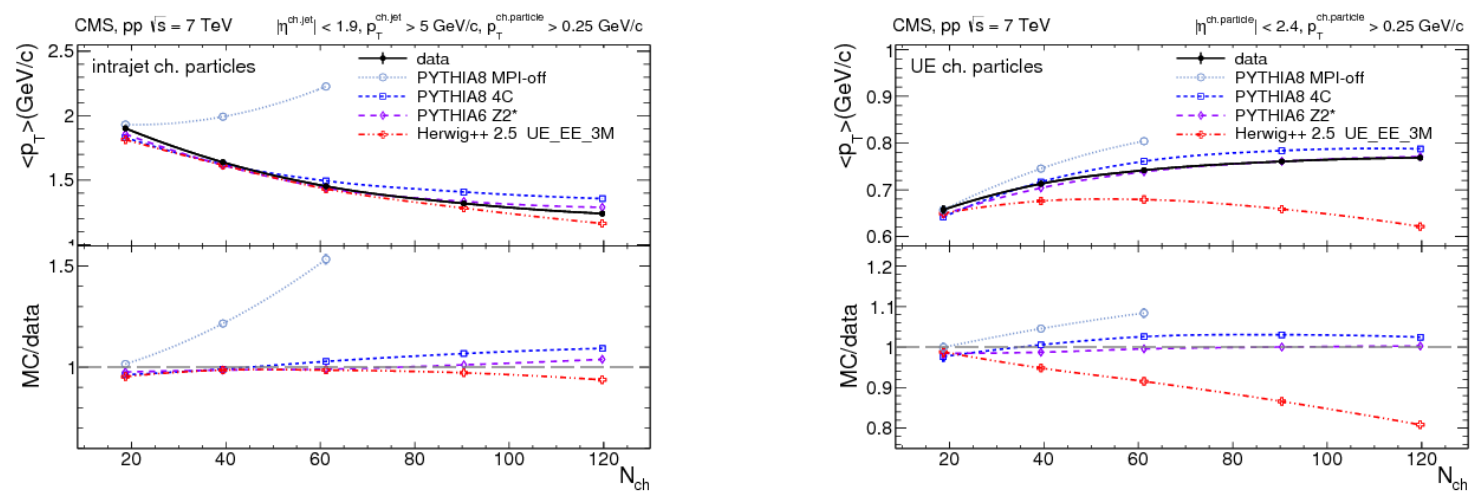

Figure 3: Comparison of the Monte Carlo predictions with the measurement of the average transverse momenta of intrajet (left) and soft (right) tracks for different particle multiplicity domains.
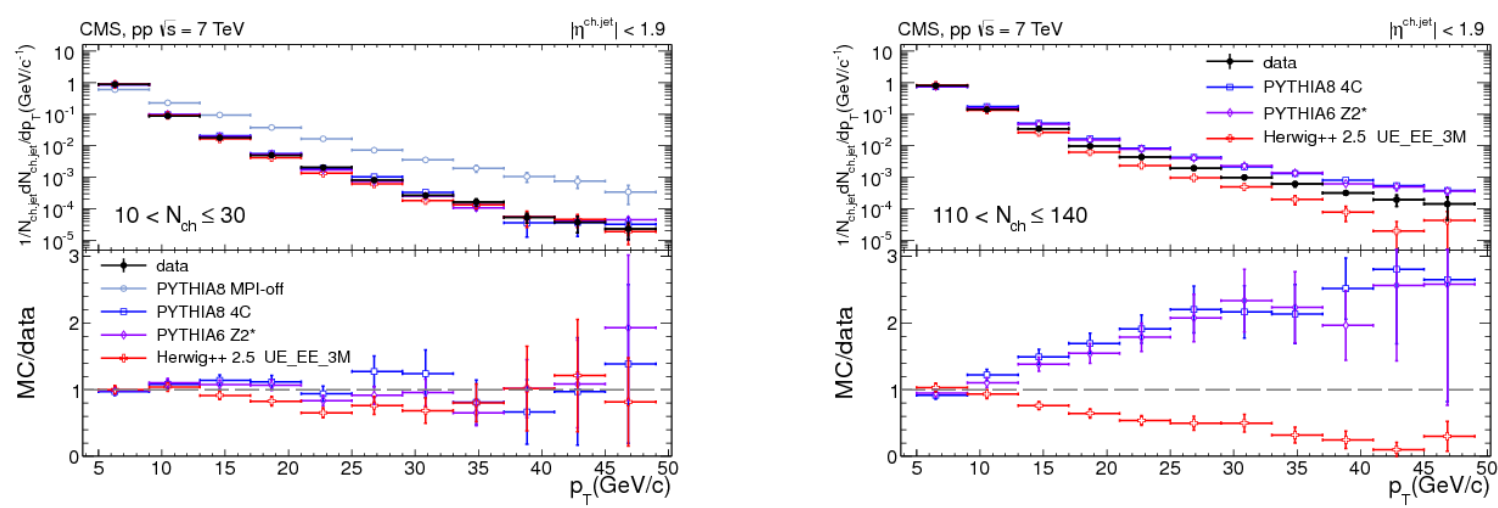

Figure 4: Comparison of the Monte Carlo predictions with the measurement of the reconstructed jet transverse momenta for the lowest (left) and the highest (right) multiplicity domains.

distributions were corrected for detector effects.

Fig. 3 shows the comparison of the mean transverse momenta of the intrajet and the soft tracks for data and different MC models for different multiplicity regions. Predictions from both PYTHIA versions (tunes 4C and Z2*) agree well with observed distributions, while for Herwig++ large differences are observed for soft tracks. The prediction without MPI is far away from the measurement showing the importance of the MPI modeling.

The jet transverse momentum distributions for the highest and the lowest multiplicity domains are shown on Fig. 4. The agreement of the MC predictions with the measurement is satisfactory in the lowest multiplicity domain for all models apart of the PYTHIA8 MPI off model. For the highest multiplicity domain the predictions from all models do not describe the measurement - the rate of hard jets is largely overestimated by both PYTHIA versions and underestimated by Herwig++.

\section{Underlying Event properties in events with $t \bar{t}$ quark pair}

The UE is also studied in events containing a $t \bar{t}$ quark pair [10], where the reference object is defined as the reconstructed momentum of the $t \bar{t}$ pair. In order to select events in which the $t \bar{t}$ 
quark pair was produced a presence of pair of isolated leptons $(e e, \mu \mu$ or $e \mu)$ with $|\eta|<2.4$ and $p_{\mathrm{T}}>20 \mathrm{GeV}$ is required.

For all events the anti- $k_{T}$ algorithm with a distance parameter of 0.4 is used. To further enrich the purity of the selected sample, events are required to have two jets with $|\eta|<2.4, p_{\mathrm{T}}>20 \mathrm{GeV}$ and passing the b-tagging criteria.

The momentum of the $t \bar{t}$ quark pair is reconstructed by summing the momenta of the t quark decay products - 2 leptons, 2 b-tagged jets and missing transverse momentum (which provides information on the momenta of neutrinos present in the t quarks decay chains). The direction in the transverse plane the $t \bar{t}$ quark pair is used to define the towards, away and transverse regions. The analysis was performed using data recorded in 2012 at $\sqrt{s}=8 \mathrm{TeV}$. The measurements are compared to predictions obtained with Madgraph [11] (interfaced to PYTHIA6 with Z2* tune for parton showering and hadronization). Distributions of the total transverse momentum flux, number of charged particles and the mean transverse momentum carried by each charged particle are shown in Fig.5. Good agreement between data and MC is observed, with slightly higher multiplicities predicted for the away region by the $\mathrm{MC}$ around $N_{c h}=40$.

\section{Summary}

Recent measurements of the underlying event activity performed with the CMS detector were summarized. Comparisons of the basic UE event properties, like total transverse momentum flux or average charged particles multiplicities in the transverse region (as a function of the interaction scale) show a good agreement with most recent UE models. More detailed studies, such as measurement of strange particle production properties in the UE or studies of the UE properties as a function of event centralities show tensions for all tested MC generators.

The measurement of the Underlying Event properties in events with a $t \bar{t}$ quark pair was in good agreement with the predictions obtained with Madgraph interfaced to PYTHIA6 (using Z2* UE tune).

\section{Acknowledgments}

I wish to thank the organizers of DIS 2014 in Warsaw for the preparation of this interesting workshop, as well as my colleuges from DESY CMS group for useful discussions and creating a fun, friendly and stimulating work environment.

\section{References}

[1] R.D.Field, "The Underlying Event in Hard Scattering Processes”, arXiv:hep-ph/0201192

[2] The CMS Collaboration, "Measurement of the underlying event activity in pp collisions at the LHC using leading tracks at $\sqrt{s}=7 \mathrm{TeV}$ and comparison with $\sqrt{s}=0.9 \mathrm{TeV}$ ”, CMS PAS FSQ-12-020

[3] M. Bähr et al., Herwig++ physics and manual, Eur. Phys. J. C 58, 639 (2008), doi:10.1140/epjc/s10052-008-0798-9

[4] S. Gieseke et al., Herwig ++ 2.5 release note (2011), arXiv:1102.1672 

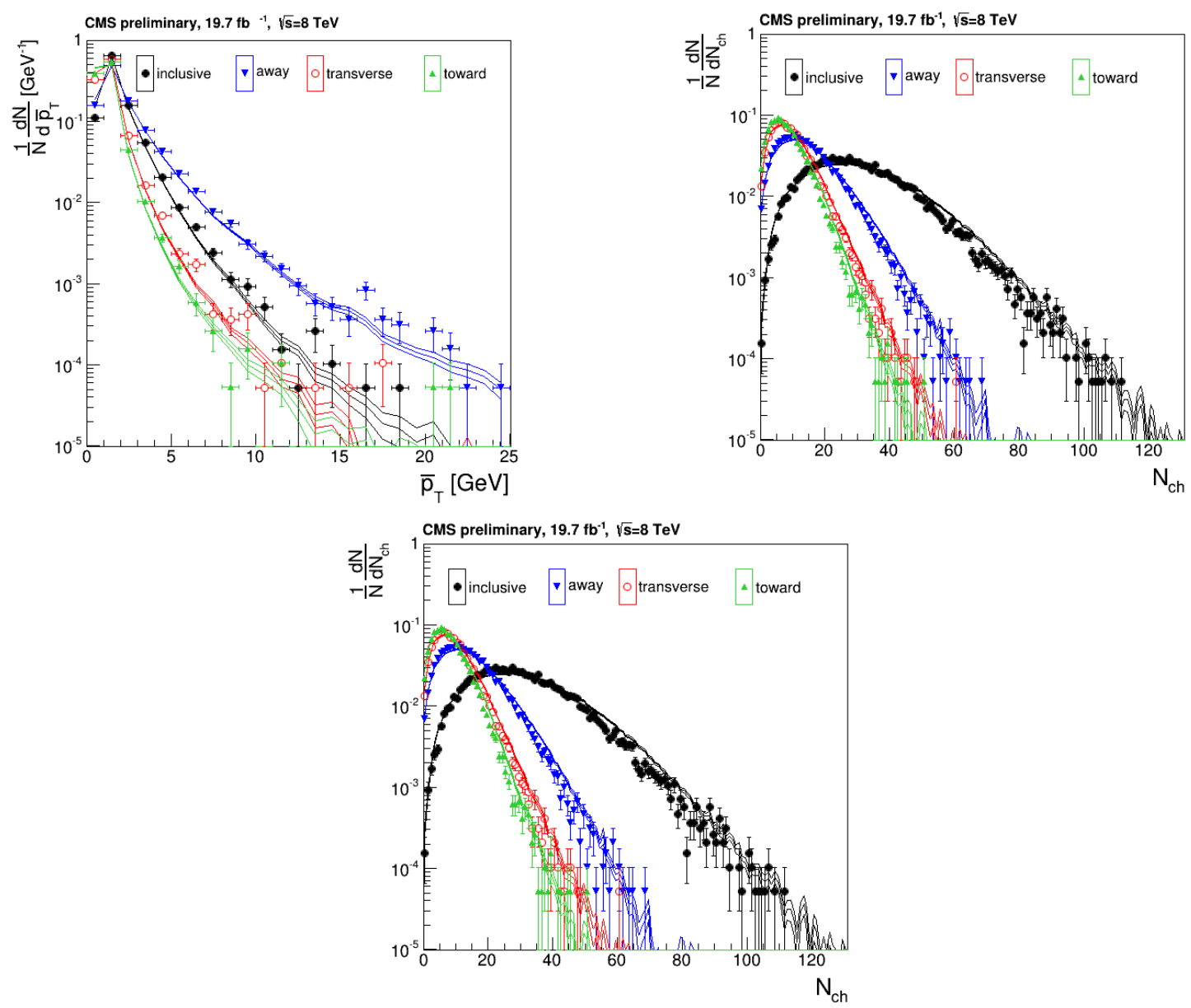

Figure 5: Total transverse momentum flux (top left), number of charged particles (top right) and mean transverse momentum carried by each charged particle in the towards, away and transverse region in events with $t \bar{t}$ quark pair.

[5] T. Sjöstrand, S. Mrenna, P. Skands, "PYTHIA 6.4 physics and manual”. J. High Energy Phys. 05, 026 (2006), doi:10.1088/1126-6708/2006/05/026

[6] T. Sjöstrand, S. Mrenna, P. Skands, "A brief introduction to PYTHIA 8.1”, Comput. Phys. Commun. 178, 852 (2008), doi:10.1016/j.cpc.2008.01.036

[7] Paolo Gunnellini, 'Measurement of four-jet production in proton-proton collisions at $\sqrt{s}=7 \mathrm{TeV}$ and UE tunes and double parton scattering”, this proceedings; also CMS PAS-GEN-14-001

[8] The CMS Collaboration, "Measurement of Strange Particle Production in Underlying Events in proton-proton collisions at $\sqrt{s}=7$ TeV", Phys.Rev. D88 (2013) 052001

[9] The CMS Collaboration, "Study of the properties of hard and soft particle production as a function of particle multiplicity in p-p collisions at $\sqrt{s}=7 \mathrm{TeV}$ ", Eur.Phys.J. C73 (2013) 2674

[10] The CMS Collaboration, "Study of the underlying event, b-quark fragmentation and hadronization properties in $t \bar{t}$ events", CMS PAS TOP-13-007

[11] Alwall et al., "MadGraph 5 : Going Beyond", JHEP 1106 (2011) 128, doi:10.1007/JHEP06(2011)128, arXiv:1106.0522. 